\title{
NMR Crystallography Advancements for Exploring Polymorphism
}

\author{
Maria Baias ${ }^{1}$, Renny Mathew ${ }^{1}$, Ivan Sergeyev², Karolina Uchman ${ }^{1}$, Melanie Rosay ${ }^{2}$, \\ Fabien Aussenac ${ }^{3}$, Werner Maas ${ }^{2}$, Chris J. Pickard ${ }^{4}$, Bart Kahr ${ }^{5}$ \\ ${ }^{1}$ Division of Science, New York University Abu Dhabi, P.O. Box 129188, Abu Dhabi, UAE \\ ${ }^{2}$ Bruker BioSpin, 15 Fortune Drive, Billerica, Massachusetts, USA \\ ${ }^{3}$ Bruker BioSpin, 34 rue de l'industrie, 67166 Wissembourg, France \\ ${ }^{4}$ University of Cambridge, UK \\ ${ }^{5}$ Chemistry Department, New York University, New York, USA \\ Email Contact: maria.baias@nyu.edu
}

The development of NMR crystallography, a method that employs a combined experimental and computational approach for structural elucidation of powdered crystalline materials, opened new paths into exploring pharmaceutical polymorphism $[1,2]$. Solid-state NMR investigation of pharmaceutical drugs is faced with two main drawbacks: the low sensitivity of NMR experiments involving nuclei such as ${ }^{13} \mathrm{C},{ }^{15} \mathrm{~N}$ at natural abundance and long ${ }^{1} \mathrm{H}_{1}$ relaxation times of many pharmaceuticals. The consequence of these cumulative effects is a very long experimental time for signal averaging required to obtain sufficiently high signal-to-noise ratio in two-dimensional NMR spectra, which are essential for the unambiguous chemical shift assignment of the investigated structure.

Our research is centered on further developing NMR crystallography by using new experimental and computational techniques that are more appropriate for studying complex molecular crystals. Experimentally we make use of the developments in Dynamic Nuclear Polarization (DNP)-enhanced solid-state NMR [3] to reduce the experimental time, allowing us to tackle more complex structures. Computationally we focus on tailoring the $A b$ Initio Random Structure Searching (AIRSS) [4] protocol to establish a DFT-based crystal structure prediction method for predicting new polymorphs of organic molecular crystals.

References

1. Baias, M. et al. (2013). Phys. Chem. Chem. Phys., 15, 8069-8080.

2. Baias, M. et al. (2013). J. Am. Chem. Soc., 135, 17501-17507.

3. Rosay, M. et al. (2016) .J. Magn. Reson., 264, 88-98.

4. Pickard, C.J.\& Needs, R.J. (2011) J. Phys.: Condens. Matter., 23, 053201. 